\title{
Optimisation of multi-body fishbot undulatory swimming speed based on SOLEIL and BhT simulators
}

\section{S. Raja Mohamed*}

\author{
KITCBE, \\ MS University, \\ Tirunelveli 627 012, TN, India \\ Email: raja_delip@yahoo.com \\ *Corresponding author

\section{P. Raviraj} \\ Department of CSE, \\ GSSSITEW, \\ Mysuru 570016, KA, India \\ Email: drpraviraj@gmail.com
}

\begin{abstract}
Robotic fish design is an upcoming and interesting research area with lot of challenging tasks due to the impulsive dynamics of water space. In this paper an evolutionary computational approach is performed to design caudal fins under carangi form and sub-carangi form swimming modes. Size and Shape with SOLEIL and multi-body evolutionary experiments were carried out using Euler-Lagrangian equation-based BhT tool to experiment and validate the hydrodynamic effects of caudal fin by avoiding complex and time consuming CFD simulations to achieve realistic motion. To improve average velocity of robotic fish two approaches have been suggested, one is a hill climbing algorithm to find optimal shape with standard stiffness whereas the second approach considers both shape and stiffness together in a genetic algorithm. Finally simulated fin models are compared against physical models to identify the correlation and performances of both to accurately approximate real world performances in a simulated environment leading to design optimised caudal fins for robotic fish.
\end{abstract}

Keywords: bio-hydrodynamics; carangiform; body-caudal fin; fin flexibility; multi-body segments.

Reference to this paper should be made as follows: Raja Mohamed, S. and Raviraj, P. (2020) 'Optimisation of multi-body fishbot undulatory swimming speed based on SOLEIL and BhT simulators', Int. J. Intelligence and Sustainable Computing, Vol. 1, No. 1, pp.19-31.

Biographical notes: S. Raja Mohamed is currently pursuing his $\mathrm{PhD}$ at MS University, Tirunelveli and completed his PG and UG in CSE from the MK and AU. His research interest includes robotics, algorithms and soft computing applications. 
P. Raviraj is currently working as a Professor of CSE at GSSS, Mysuru and received his $\mathrm{PhD}$ from the VMU a decade ago. His research interest includes image processing and computer vision algorithms and currently guiding several scholar is image mining related research project.

\section{Introduction}

Motivated by Mother Nature, robotic experts have designed models as adept and capable as natural fish swimming. However bio-mimetic robots are not as skilful as their biological equivalents due to the resources involved in building robotic fish to work similar to organic tissue. Yet, robotic fish has several benefits when compared with propeller-driven underwater vehicles. First, with few moving components and extra space for sensors with reduced power consumption. Then, a real-life like exterior may be pushing the system in to its natural bionetwork in terms of appearance. Given uniqueness such as above, it has been extended in environmental monitoring to biological life study applications.

The key problem in developing robotic fish can be the field vagueness, as the marine environment is exceedingly nonlinear, with highly demanding and having complicated design process. Because of this, hydrodynamic interactions performed with mathematical models tested in such environments can provide better design process by means of test design. Still with an ideal numerical model, the design process hangs with dispute in terms of bulky number of parameters concerned towards sensible motion. Electro-mechanical components and their materials with constraints will have different performances and also requires information regarding the properties of materials used. To construct a flexible caudal fin elasticity coefficient of material must be known and it would be fine to have an automated design process capable of handling high-dimensionality. Evolutionary computational techniques are well suited for problems with high dimensionality where the solution space is sampled to bring certain aspects of exclusive solutions in creating efficient mixtures. To identify effective and novel solutions we can integrate mathematical model during evaluation phase to track the peculiarities of an aquatic environment. These kinds of solutions allow the designers to have complete idea about excellent robotic fish design.

Here we put forward an evolutionary method to design caudal fin of a robotic fish. Optimisation takes place in a rigid multi-body dynamics using mathematical model with respect to hydrodynamics of linked caudal fin. Then solutions which were simulated are compared to predictions brought by mathematical model in such a way that the stiffness coefficient of a fin with fixed shape is optimised using hill-climber algorithm, and the fitness landscape is also compared against the derived model. To validate the results, set of fins are made and then tested over robotic fish prototype in an aquatic environment. To finish the optimisation process involving physical characteristics of the caudal fin an optimisation algorithm is used which specifically deals with stiffness and dimensions of caudal fin concurrently evolved against given control pattern. The task is about deploying evolutionary design involving present-day dynamic models which can be personalised into all-purpose engineering process under robotics. 


\section{Background work}

Robotic fish has plenty of applications such as studying natural fish morphology and behaviour at fish aquarium and environmental monitoring such water pollution, etc. It allows the researchers to reproduce with control to evaluate the behaviour of real fish Faria et al. (2010), or in learning natural evolution and other biological assumptions referred by Long et al. (2011, 2006). More recently Marras and Porfiri (2012) and Raja Moahemd and Raviraj (2015) robotic fish have shown that tethered robot with moving caudal fin able to form schooling behaviour similar to natural fish inside tank. When the tail fin was not moving, then natural fish did not react for schooling to support the assumptions that a bio-mimetic robot can aid in reading behavioural aspects. As vindicated by the preceding one, fish can act together with a realistic robot like a natural fish. With more and more refined designs, insight into fish actions can be taken to solve problems by observing biological fish in natural or a fixed lab atmosphere stated in Raja Moahemd and Raviraj (2011). At the same time robotic fish was also used to observe underwater activities like oil spill surveying detection, etc. by Wang et al. (2011). As it appears similar to natural fish mobile sensor can be placed to observe ecosystems without interference.

Bio-inspired technique in the sense design and fabrication of fin extracted from nature for modelling in accordance to nature stated by Masoomi et al. (2013). Two major types of swimming locomotion (anguilliform, carangiform) require specific mathematical model to exactly illustrate the dynamics of motion. A ribbon-like fin with a cord of actuators coupled by soft material has been shown and also capable of replicating the force of real fins used by Epstein et al. (2006). Further, research work of Chen et al. (2010), Hu et al. (2009) and Tan et al. (2010) gives insight into carangiform locomotion, where the forward thrust is mainly generated by the tail or caudal fin. Recently, a mathematical model was proposed to cover multiple aspects of locomotion pertaining bendable carangiform mode of swimming (Wang et al., 2011).

Virtual creatures created based on Morphological evolution have been the focus in bringing real outlook. They key obstacle in any simulation-developed solution is about transformation of physical robot from data. There is a reality-gap for solutions that are working well during simulation may face issues such as improper modelling or out of the blue glitches in physical environment. To deal this problem, Bongard and Lipson (2004) simulator model is evolved concurrently with robotic fish and openly accepting solutions for similar performances in reality by comparing with simulation listed in Swarnamugi et al. (2016). In the latter, solutions having higher transferability are considered as fit. To narrow the gap further, accurate models are deployed for exclusive environmental conditions. Earlier, Gomez and Miikkulainen (2003), for instance, they demonstrated that an exhaustive simulator working in tandem alongside evolutionary algorithm to produce controllers for finless rockets operating in dynamic environment. In the recent past reality gap extended to contain material properties and their reaction to explicit environmental situation pointed by Saxena and Chauhan (2017).

As a fact that modelling such connections at the very low level are at the moment is difficult. Here the approach is to put together evolutionary computation and mathematical modelling of material with properties. Evolutionary computation steers the overall procedure; engineering is required to model behaviour of essential resources when 
dynamic forces are applied, enabling near perfect assessment of the robot during simulation according to Anu Priya et al. (2015).

\section{Methodology}

SOLEIL is an extended integral equation solver for shape and size optimisation. Then BhT to simulate vortex free rigid-body dynamics based on ordinary differential equations to analyse the interaction multi-body segments with fluid. In addition, to guarantee the significance of results it was tested in SOLEIL against fins that were tested on a robotic fish prototype with varying number of segments from 1 to 3 .

\subsection{Mathematical model}

With the help of rigid-body dynamics, movement of caudal fin can be estimated by dividing the flexible fin into discrete segments coupled by relays with flexible movement. Yet, the fluidic movement of a fin during locomotion is tough to model in simulation and difficult to imitate on a physical robot too. James Lighthill's (1971) theory of Locomotion for elongated body was proposed to realise fish locomotion as a sequence of flexible segments and their movement at any given point can be estimated using equations resulting thrust and movement of the same.

Figure 1 Effect of dynamic forces over passive flexible caudal fin segments (see online version for colours)

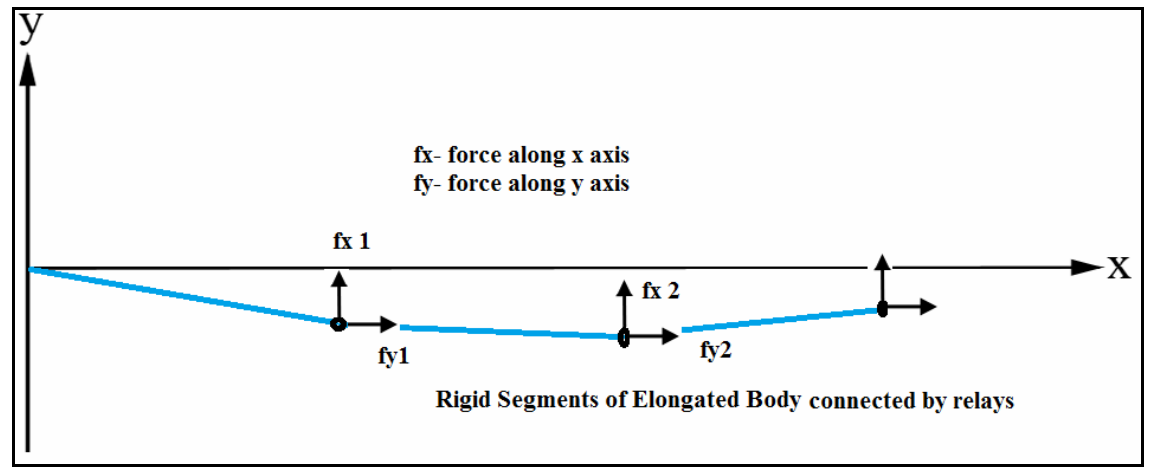

Basically three different fin shapes have been considered in our testing. To calculate the dynamic forces generated by various fins based on improved Lighthill's theory, Wang et al. (2012) divided the caudal fin segments of equal-size and evaluated forces independently against each of them by considering extra forces acting at the tip. These fin segments are assumed to be connected through a sequence of relays forming a flexible body and fin structure with respect to Lighthill's elongated body mathematical model.

In Figure 1 body segments are shown along with the forces acting against individual regions. Each segment produces couple of forces; one is a resistive and the other is propulsive component in accordance with mathematical model. Equation (1) describes the hydrodynamic force act against each segment. 


$$
f^{\prime}(t)=\left(f_{x}(t), f_{y}(t)=-m(d / d t)\left(v \perp n^{\prime}\right)\right)
$$

In equation (1), $m$ refers unit length mass, $t$ points out the position where the force acts over the fin, and $n^{\prime}$ denotes unit direction and $v$ denotes velocity at right angles to the fin. Then extra force at the tip of last segment is computed by equation (2):

$$
f_{l}^{\prime}=\left(f_{l x}, f_{l y}\right)=\left[-1 / 2 m v^{2} m^{\prime}+m v \perp v n^{\prime}\right]_{t=l}
$$

where $t=1$ represents the backside end of fin, where $m^{\prime}$ refers the unit direction, $v$ refers velocity acting parallel to fin. By giving $x$ and $y$ coordinate values of each fin segment at time to calculate hydrodynamic forces.

Each fin segment is attached to the body through relays which creates periodic motion in a sinusoidal mode. Then parameters such as amplitude, frequency are set for the same using various delay and sweep angles, including the material's dimensions, Young's modulus of elasticity to determine flexibility for the relays and joints also included in the parameter list. This correlation helps us to transfer simulated designs into physical materials using known and incidental properties.

\subsection{Suitable simulation environment}

An environment to face the unique challenges with respect to modelling the hydro dynamics of an aquatic surrounding, BhT and SOLEIL are used for 2D and 3D simulation of mathematical model to compute hydrodynamic forces at the end of caudal fin. It avoids complex and lengthy time consuming computational fluid dynamics calculations and the reduction in computational time is profitable especially in the case number of tests for best solution through simulation. The mathematical model restricts motion with in a 2D plane and assumes floating tendency without any outside forces.

A robotic fish prototype is created from simulated data, to analyse the performance of fin having various dimensions and material having different stiffness values. Virtual model is shown in Figure 2, having main and three-segment caudal fin using BhT. Fin flexibility was estimated with inactive hinges fixed between segments run by time and sweep angles of relays. It allows the fin segment to bend at different rate of speed based on time and angular range of 15 degrees on either side as programmed.

\subsection{Physical finding}

To verify the outcomes of proposed method, fins were made-up with a variety of physical materials to generate flexible moves which is similar to simulation. Three basic fin models fabricated and connected to a robotic fish for evaluation. Figure 3 shows the physical robot with one type of fin attached to it

To calculate the average velocity of each fin model time trials were used, while visual observations also help in determining the bending ability of fins during motion. During the trials, the height, length and thickness of each fin segment was fixed at 3.0, 4.0 and $0.1 \mathrm{~cm}$ respectively. The Young's coefficient of elasticity was taken from the data sheets. 
Figure 2 Virtual fish model with a three-segment rigid-body using SOLEIL (see online version for colours)

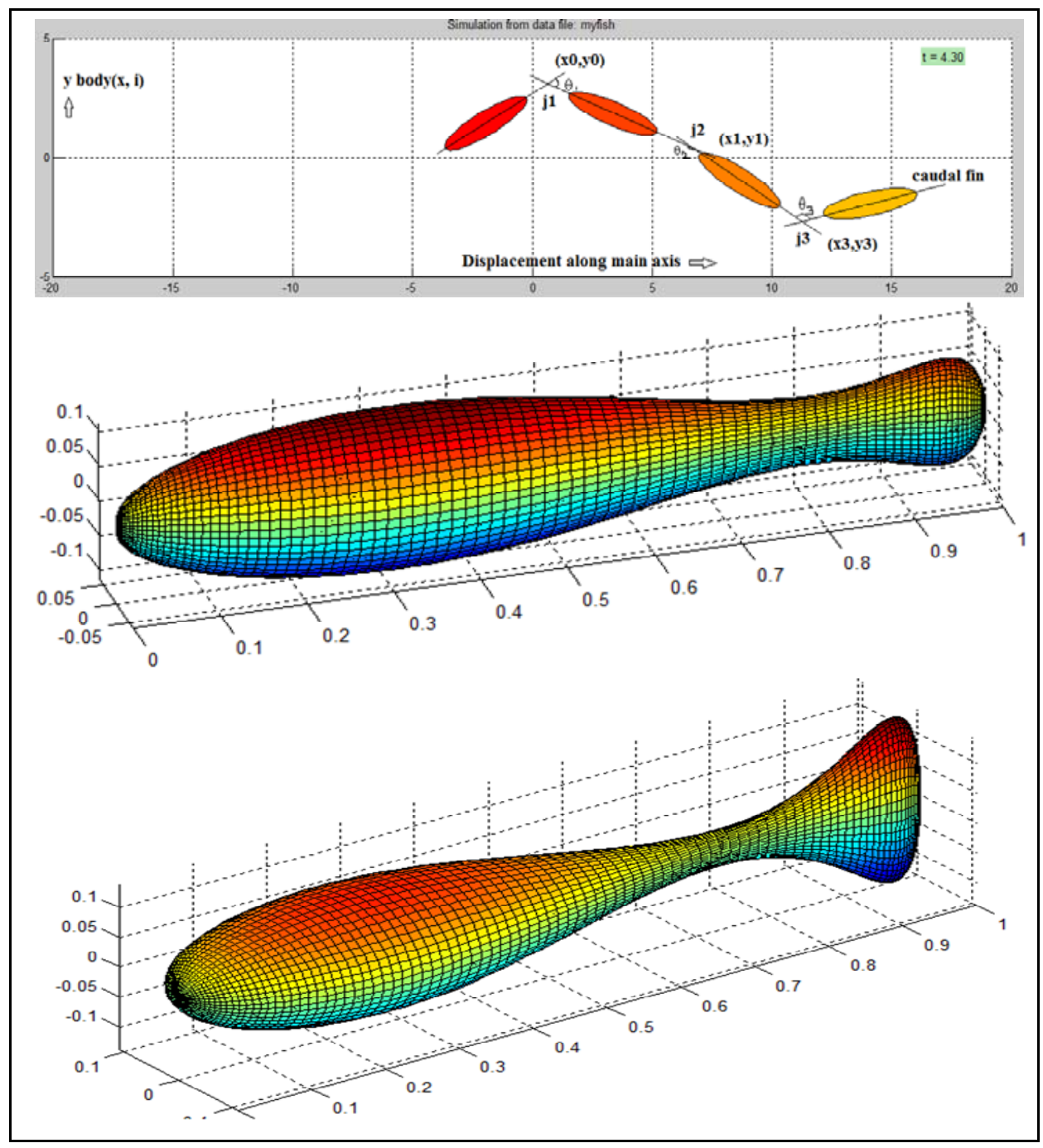

The prototype robot was positioned inside tank to reach a steady state swimming speed prior to computing average velocity using different fin size and shape. Stiffness of each fin segment is computed using equation (3):

$$
K_{s}=\left(E d h^{3} / 12 l\right)
$$

In which $K_{s}$ denotes torsion spring constant of material, $d$ refers height and $l$ refers length of the fin segment. $E$ denotes Young's coefficient of elasticity and $h$ denotes thickness of each fin segment. Obtained values from the above equation can be used straight away in simulation at some point in optimisation routines to produce an effectively equating simulation and physical results to maximum perfection level possible. 
Figure 3 Prototype robotic fish with one type of caudal fin controlled using a servo motor(s) with a specific range of motion and oscillatory sweep angles for different segments (see online version for colours)

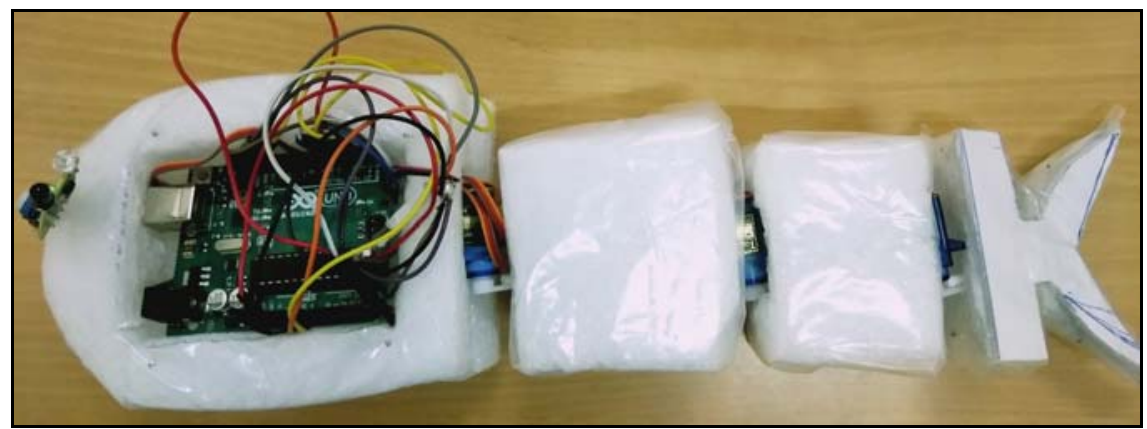

\section{Experiments and results}

This paper deals problem by dividing them in to three sections such as mathematical and physical model validation, followed by evolutionary optimisation. First simulation results were compared with data resulting from the mathematical model followed by comparison between simulation and physical data obtained from experiments were made. Finally after validating the simulation data, evolutionary computational technique was applied in designing process of flexible caudal fin.

\subsection{Comparing mathematical and simulation models}

Before proceeding to bodily validation and evolutionary experiments, we need to make sure that the simulation background should be set up for the mathematical model in achieving perfection. Any inequality between these two would imply faulty evolutionary results which are useless. By considering the above, couple of algorithms were engaged to get the optimised stiffness value for simulated system during which Young's coefficient for flexibility alone was changed for both the experiments.

Initial algorithm is a basic hill-climber with 100 independent runs and each run was started with a specific seed and Young's coefficient value selected consistently between the range $[0.5,4 \mathrm{GPa}]$ randomly. Young's modulus value was calculated by translating using Equation (3), to the joint coefficients that control caudal fin flexibility. After configuring the values of robot, it was allowed to swim for about ten seconds. Simultaneously fitness of the same for each Young's coefficient was figured to achieve average velocity during evaluation period. Every time hill-climber algorithm begins with the evaluation of the arbitrarily selected initial Young's coefficient value, succeeding values were generated by relocating the current one by a random number selected over and over again from a Gaussian distribution having mean value of 0 and variance of 0.1 . The resultant Young's coefficient value was evaluated, and the higher (better) average velocity value was set aside for the next test case to be produced. In each run, the process was iterated to have at least 100 candidate values to be evaluated. Every hill-climber occurrence converged to an optimum Young's coefficient value of more or less $1.7 \mathrm{GPa}$, 
and given some amount of time it is expected that remaining final values may converge at a single optimal value.

Second one is a formal genetic algorithm with the primary target of confirming that the simulation environment is well suited work alongside evolutionary algorithm. It has 30 free runs comprised as an experiment and each run was starting with a unique value and a collection of 100 randomly generated individuals. Each one tested similar to the one used in hill-climber test. After 100 generations, populations advance with mutation as the mere operator for evolution. Then by initialising population, successive generations were produced using three-individual contest like selection processes and a Gaussian mutation operator. In addition, to ensure holding on to better fitness value, the majority of $10 \%$ of the population was deemed as privileged and passed on to the next generation without alteration. Evolutionary experimental result closely reminds the individual best fit cases of hill-climber in each run, with Young's coefficient values around 1.6 GPa. Figure 4 shows the data obtained from mathematical model. Figure 5 shows the results of both simulation experiments in which hill climber and evolutionary approach yielding almost identical solutions. Though it is an anticipated result, both experiments need similar simulation environment. When we have a close look Figures 4 and 5, there is a difference involving model and simulation results which is very clear. In particular, the model forecasts max average velocity around $4.8 \mathrm{~cm} / \mathrm{s}$ when Young's coefficients sit around $0.7 \mathrm{GPa}$, whereas simulation results approach max average velocity closer to $1.2 \mathrm{~cm} / \mathrm{s}$ at a Young's coefficients near $0.9 \mathrm{GPa}$.

Figure 4 Mathematical model predicted velocities by assuming that the body is stable (see online version for colours)

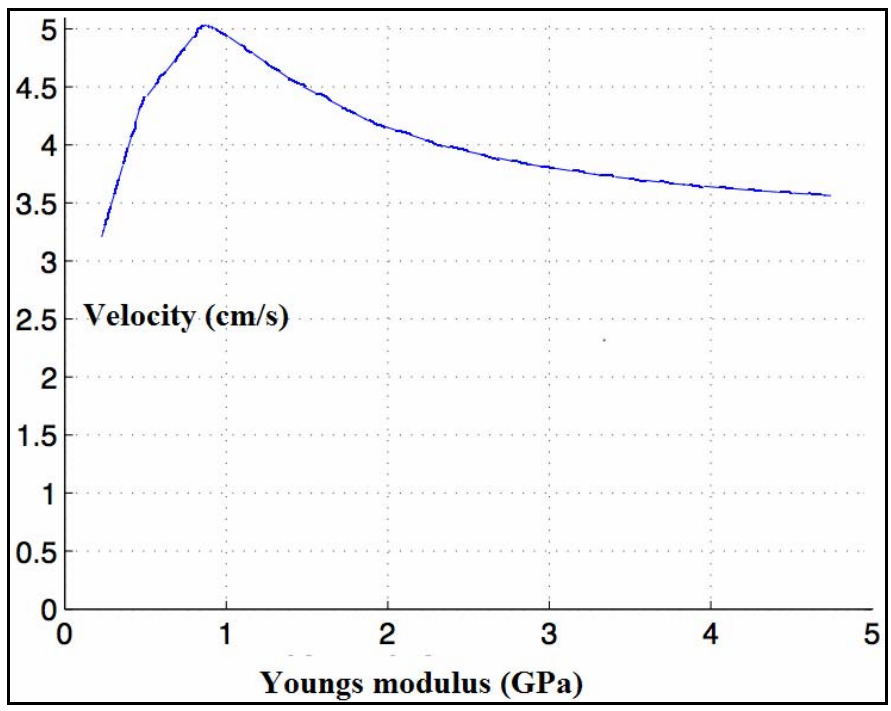

In spite of differences between both it shows the similar fashion, in which mid-way values of the Young's coefficients turn out to be best and fast robotic fish. Further, the difference between the figures examined a bit closer with model and simulator. Interestingly with an assumption that the robotic fish body having no effect over caudal fin motion according to mathematical model as well as fin segment without mass. These two assumptions are not approved in the simulation environment, and they would cause 
simulated one to look slower compared to model data prediction. In order to determine physically meaningful physical results, we need examine them in the proposed methodology.

Figure 5 Determining the optimum stiffness of a fixed dimension fin with both hill-climber and evolutionary algorithm and they converged almost on a common stiffness values offering the highest average velocity (see online version for colours)

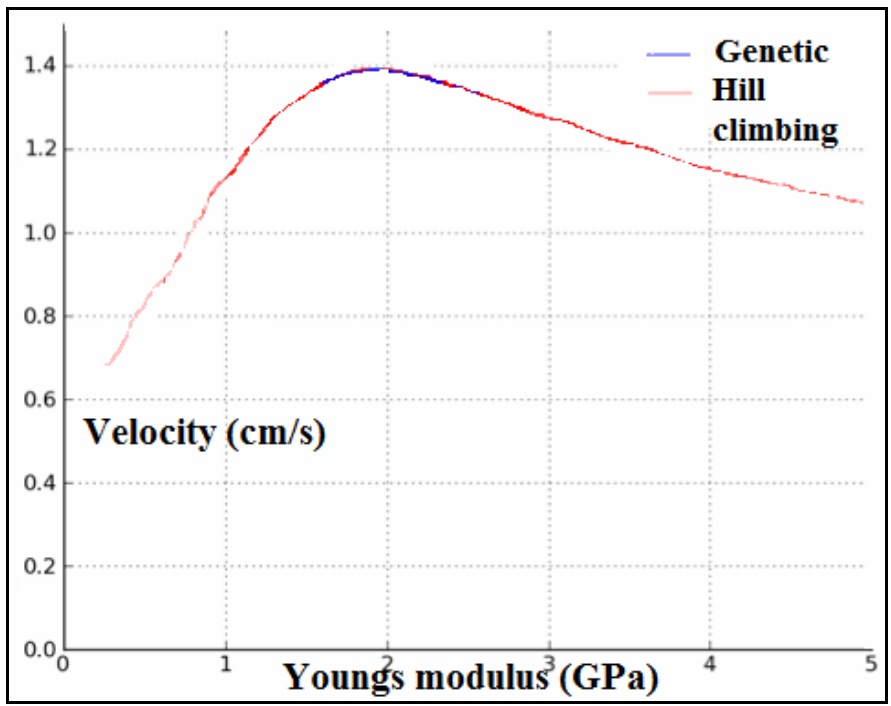

\subsection{Physical validation}

To authenticate cases taken from simulation, we have designed three different caudal fins with similar size and different shapes and tested the same with fish prototype. Unique stiffness values for fins under each model were created and the materials used were ranging from high to low flexibility. Each fin was attached to the prototype to measure the average velocity over 3 separate trials. The results of the above experiments are shown in Figure 6. Similar to the predicted values, it points out flexibility having highest average velocity in the intermediate level. But comparisons linking simulation and reality are not worthwhile due to boundaries of materials. In particular, the materials are not having specific Young's coefficients value; rather the maker offering range of likely values for each material.

Despite using fins of identical shape and size for their respective flexibility values the mathematical model, simulation, and physical data could make meaningful comparison. First, the physical and mathematical model velocity values are closer when compared to simulation results. The data composed from experiments will be helpful to improve the model and the environment of simulation. In addition, the optimal Young's coefficient range is 1-2 GPa for most of the results obtained during experiment. The reason behind the gap in the model predictions are known, however it is also obvious that there is no match between simulation and reality. The maximum velocity achieved during the physical experiments is $3.7 \mathrm{~cm} / \mathrm{s}$ is almost double that of simulation velocity. In the mathematical model estimations were made to fine tune simulation environment. 
Distributed force over the body is treated as single point force, and the fin segments are split into three parts. Fin size is decreased relatively with respect to previous segment, and increase in the number of segments, the movement and separation of forces will be more practical and expected to increase the accuracy for simulation experiments. Alternatively the simulation and physical tests were observed for flexibility of fins during oscillation to measure its performance.

\subsection{Evolution of fin structure}

Once after contrasting numerical and simulation model results, process of optimisation was extended during evolutionary computation too. During this process Young's coefficients and dimensions of a three different caudal fins were concurrently evolved. Further fin shape was permitted to evolve under the conditions that the length-height face, thickness and area o the fin remain fixed. Now the aspect ratio of the fin was not allowed to evolve and the Young's coefficient and length of a fin to evolve. Practically various fin dimensions were taken into consideration with max length of $14 \mathrm{~cm}$ and min length of $4 \mathrm{~cm}$ forced during evolution. Anything that lies outside this range will have transferability issues with respect electromechanical constraints. Altogether 30 repeated test runs were performed to identify the correlation between parameters such as stiffness, shape, and average velocity of caudal fin.

Figure 6 Average velocity for calculate for materials used to fabricate fins and flexibitiy decreases for towards $\mathrm{x}$ axis (see online version for colours)

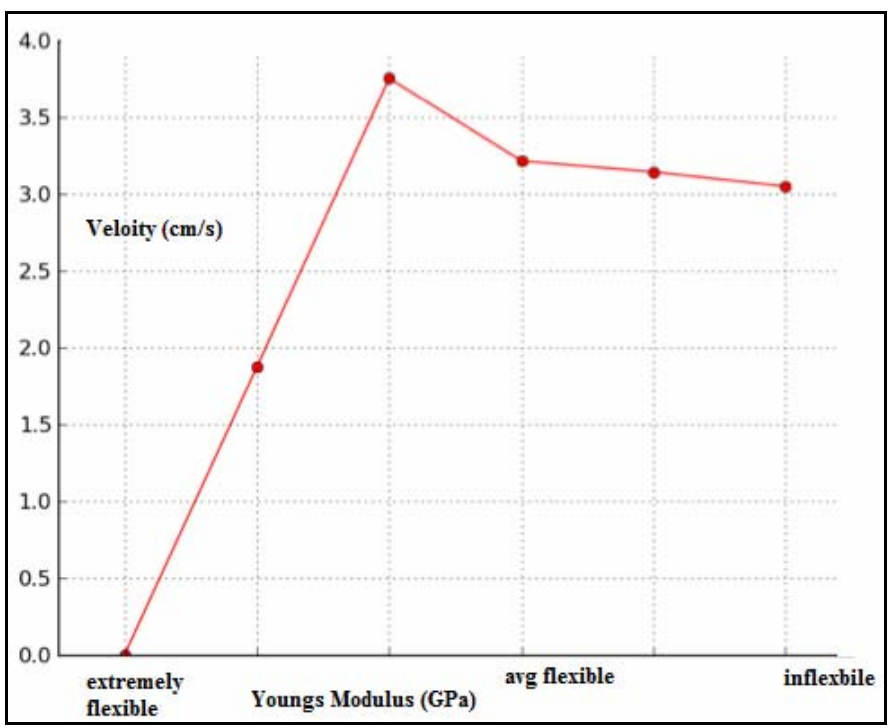

Optimum values in a set were found for both Young's coefficient and dimensions of the fin from the evolutionary runs performed earlier in which Young's coefficient found during trial run was $7.55 \mathrm{GPa}$, at which length and height of caudal fin was $14 \mathrm{~cm}$ and $1.43 \mathrm{~cm}$ respectively. Consequently, the best solution reached the max fin length allowed with respect to fin width. As we expected such a result, longer the fin length higher the propulsive force, where as the fin width has lesser effect over the same. Equation (2) 
exhibits the same property, where the longer fins having higher angular velocity close to the posterior part of fin as we increase the length in linear fashion.

Interestingly Young's coefficient value found during trial is larger than prior experiments, accordingly the stiffness of material is around $1.35 \times 10^{-3} \mathrm{Nm}$ for non trial cases, and $1.73 \times 10^{-3} \mathrm{Nm}$ for complete evolution based experiments. It also implies that using a constant stiffness value may be enough for varying caudal fin dimensions and shapes. Further, stiffness values are alike when the length is increased with the same done for Young's coefficient to maintain rather than constant value. Here, Figure 7 depicts 3D fitness landscape obtained during the evolutionary experiment. Its peak is sited at a coefficient of elasticity around $7.5 \mathrm{GPa}$ with $14 \mathrm{~cm}$ of length. These set of values produced an average velocity of $2.2 \mathrm{~cm} / \mathrm{s}$ as Figure 7 suggests that for every set of dimensions there is a precise Young's coefficient value that is associated with ultimate performance for a fin.

Figure 7 Fitness landscape for various shape and stiffness values of caudal fins (see online version for colours)

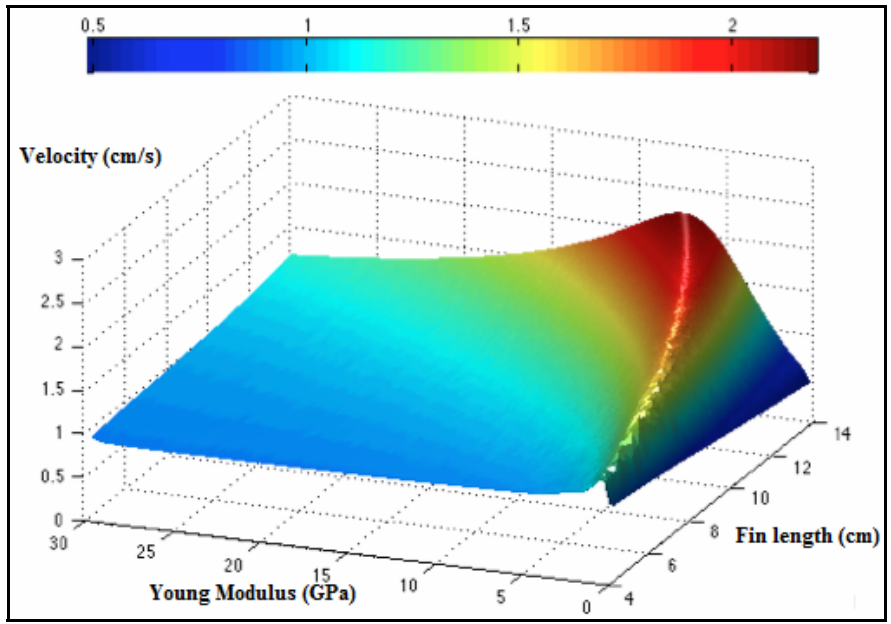

Notes: The height relies on length while determining shape, thus, height is ignored. When the fin length increases, the Young's coefficient values also increases in order to preserve similar stiffness fins for different lengths.

Highly challenging dynamics of an aquatic environment is making the design of robotic fish as demanding engineering attempt. Considering the complexity, it is really important to deploy design process that is automatic in choice using which parameters of fins can be optimised for exclusive cases and tasks.

Adapting the hydrodynamic model for caudal fin design using automated simulation process helps in optimising Young's coefficients determining the flexibility. During simulation optimum Young's coefficient mainly relies on motion and dimension of fins. For a given set of attributes including frequency of fin sweep, amplitude, height, width and length there is a unique Young's coefficient value being optimum. When the coefficient value was evolved together with fin shape, it found to be worthy as the values of length and Young's coefficient having higher and producing faster swimming caudal fins. 


\section{Conclusions}

Here we have proposed an evolution based process to design caudal fin with the help of simulation tools, i.e., BhT and SOLEIL with suitable modification in which variety of configurations for fin to be tested. The simulation part uses mathematical model and rigid-body dynamics engine for flexible caudal fin's hydrodynamics exclusively in an aquatic environment. To test the above, first hill-climber algorithm was used with a specific fin shape and control pattern, to find fitness region against stiffness and velocity. The results were compared to data produced by model, which helped us in confirming the simulation and the model with equivalent dynamics despite the absolute values seem to be different. Graph plotted against stiffness and velocity for simulation and mathematical model experiments have similar development in which average velocity was more for mid-way caudal fin flexibility is shown in fig.6. It also proves that certain important aspects related dynamics of fin can be captured for various materials. Then progression was made from hill-climber experiments to an evolutionary algorithm to optimise several fin parameters. Traditional genetic algorithm was in place to advance both the Young's coefficient and shape of fins. After performing series of respective experiments, best fit fins happen to have lengthy fins with literally constant stiffness. Hence it is obvious that longer fins usually offer large amount of force towards propulsion. In addition, it also proved that each fin shape and control pattern has its own optimal Young's coefficient value. The results obtained from simulation and physical experiments discussed here show the effectiveness of an evolutionary based approach with dimensionality. Still the work is not over as we can focus on improving the overall design process. Here assumptions related to the hydrodynamic model can be ignored say, the body segment will not be considered as static and the fins without mass followed by gradually reduce the constraints placed on evolution. Ultimately, several features of the robot can be evolved in a process which can be generalised to any non-linear robotic environment.

\section{References}

Anu Priya, A., Raja Mohamed, S. and Raviraj, P. (2016) 'A survey on various robotic fish models based on oscillatory motion', International Journal of Computer Applications, Vol. 142, No. 2, pp.37-42.

Bongard, J.C. and Lipson, H. (2004) 'Once more unto the breach: co-evolving a robot and its simulator', in Proceedings of the Ninth International Conference on the Simulation and Synthesis of Living Systems, Boston, Massachusetts, USA, pp.57-62.

Chen, Z., Shatara, S. and Tan, X. (2010) 'Modeling of biomimetic robotic fish propelled by an ionic polymer metal composite caudal fin', IEEE/ASME Transactions on Mechatronics, Vol. 15 , No. 3, pp.448-459.

Epstein, M., Colgate, J. and MacIver, M. (2006) 'Generating thrust with a biologically-inspired robotic ribbon fin', in Proceedings of the 2006 IEEE/RSJ International Conference on Intelligent Robots and Systems, Beijing, China, pp.2412-2417.

Faria, J., Dyer, J., Clement, R., Couzin, I., Holt, N., Ward, A., Waters, D. and Krause, J. (2010) 'A novel method for investigating the collective behaviour of fish: introducing 'Robofish", Behavioral Ecology and Sociobiology, Vol. 64, No. 8, pp.1211-1218.

Gomez, F.J. and Miikkulainen, R. (2003) 'Active guidance for a finless rocket using neuroevolution', in Proceedings of the 2003 Genetic and Evolutionary Computation Conference, Morgan Kaufmann, San Francisco, California, USA, pp.2084-2095. 
Hu, Q., Hedgepeth, D., Xu, L. and Tan, X. (2009) 'A framework for modeling steady turning of robotic fish', in Proceedings of the 2009 IEEE International Conference on Robotics and Automation, Kobe, Japan, pp.2669-2674.

Lighthill, M.J. (1971) 'Large-amplitude elongated-body theory of fish locomotion', in Proceedings of the Royal Society of Londo', Series B, Biological Sciences, Vol. 179, No. 1055, pp.125-138.

Long, J.H., Koob, T.J., Irving, K., Combie, K., Engel, V., Livingston, N., Lammert, A. and Schumacher, J. (2006) 'Biomimetic evolutionary analysis: testing the adaptive value of vertebrate tail stiffness in autonomous swimming robots', Journal of Experimental Biology, Vol. 209, No. 23, pp.4732-4746.

Long, J.H., Krenitsky, N.M., Roberts, S.F., Hirokawa, J., de Leeuw, J. and Porter, M.E. (2011) 'Testing biomimetic structures in bioinspired robots: how vertebrae control the stiffness of the body and the behavior of fish-like swimmers', Integrative and Comparative Biology, Vol. 51, No. 1, pp.158-75.

Marras, S. and Porfiri, M. (2012) 'Fish and robots swimming together: attraction towards the robot demands biomimetic locomotion', Journal of The Royal Society Interface, Vol. 9, No. 73, pp.1856-1868.

Masoomi, S.F., Gutschmidt, S. and Chen, X. (2013) 'Efficiency-based optimization of a 2 DOF robotic fish model', International Journal of Biomechatronics and Biomedical Robotics, Vol. 2, No. 4, pp.93-101.

Raja Moahemd, S. and Raviraj, P. (2011) 'Biologically inspired design framework for robot in dynamic environments using framsticks', International Journal on Bioinformatics and Biosciences, Vol. 1, No. 1, pp.27-35.

Raja Moahemd, S. and Raviraj, P. (2015) 'Bio-inspired swimming techniques for robotic fish using flow and pressure sensing mechanism (computational bio-mimetic)', Indian Journal of Science and Technology, Vol. 8, No. 24, pp.1-10.

Raja Moahemd, S. and Raviraj, P. (2018) 'Optimized curve fitting for multi-body segments using bio-hydrodynamics simulator', International Journal of Computer Sciences and Engineering, Vol. 6, No. 6, pp.123-128.

Saxena, M. and Chauhan, N.R. (2017) 'A review study on bioinspired robotic fish', International Journal of Mechanisms and Robotic Systems, Vol. 4, No. 1, pp.1-23.

Swarnamugi, S., Raja Mohamed, S. and Raviraj, S. (2016), 'A survey on undulatory motion-based robotic fish design', Computer Applications an International Journal, Vol. 3, No. 1, pp.1-10.

Tan, X., Carpenter, M., Thon, J. and Alequin-Ramos, F. (2010) 'Analytical modeling and experimental studies of robotic fishturning', in Proceedings of the 2010 IEEE International Conference on Robotics and Automation, Anchorage, Alaska, USA, pp.102-108.

Wang J, Alequin-Ramos F, Tan X. (2011) 'Dynamic modeling of robotic fish and its experimental validation', in Proceedings of the 2011 IEEE/RSJ International Conference on Intelligent Robots and Systems, San Francisco, California, USA, pp.588-594.

Wang, J., McKinley, P.K. and Tan, X. (2012) 'Dynamic modeling of robotic fish with a flexible caudal fin', in Proceedings of the 5th Annual Dynamic Systems and Control Conference, Ft. Lauderdale, Florida, USA. 